NASA-CR-202628

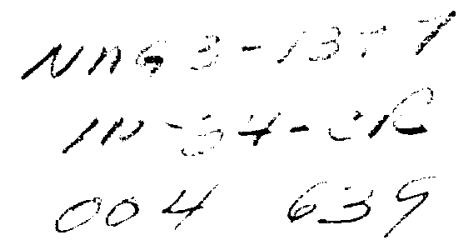

\title{
Interfacial Force Field Characterization in a Constrained Vapor Bubble Thermosyphon
}

\author{
Sunando DasGupta, Joel L. Plawsky, and Peter C. Wayner, Jr. \\ The Isermann Dept. of Chemical Engineering, Rensselaer Polytechnic Institute, Troy, NY 12180
}

\begin{abstract}
Isothermal profiles of the extended meniscus in a quartz cuvette were measured in the earth's gravitational field using an image-anatyzing interferometer that is based on computer-enhanced video microscopy of the naturally occuming interference fringes. These profiles are a function of the stress field. Experimentally, the augmented Young - Laplace equation is an excellent model for the force field at the solid-liquid-vapor interfaces for heptane and pentane menisci on quartz and tetradecane on SFL6. Effects of refractive indices of the solid and liquid on the measurement techniques were demonstrated. Experimentally obtained values of the disjoining pressure and dispersion constants were compared to those predicted from the Dzyaloshinskii-Lifshitz-Pitaeuskii theory for an ideal surface and reasonable agreements were obtained. A parameter introduced gives a quantitative measurement of the closeness of the system to equilibrium. The nonequilibrium behavior of this parameter is also presented.
\end{abstract}

\section{Introduction}

In thin liquid films, the shape-dependent interfacial stress field controls fluid flow and heat transfer (Derjaguin et al., 1965; Huh and Scriven, 1971; Potash and Wayner, 1972; Miller and Ruckenstein, 1974; Wayner et al., 1976; Teletzke et al., 1987; de Gennes, 1985; and Wayner. 1991). These concepts are particularly useful under microgravity conditions where the interfacial force field can predominate in systems much larger than those in the earth's gravitational field. When the gravitational body force is essentially removed, the shape of a constrained liquid volume with a free interface in a container changes dramatically to reflect the new force field. The resulting equilibrium shape under microgravity conditions depends on the intermolecular force field that changes rapidly in the vicinity of the liquid-vapor and liquid-solid interfaces. This liquid film shape is controlled by fixing the container shape, the liquid and solid substrate surface properties, and the volume of the liquid. Under nonequilibrium conditions, microgravity fluid dynamics and change of phase heat transfer are a function of further changes in the shape of the fluid volume from its equilibrium shape. Herein, we discuss the results of ground-based equilibrium experiments that are a precursor to nonequilibrium microgravity experiments. We

Correspondence concerning this article should be addressed to $P$. C. Wayner. Ir Cortespondence concerning of Technology. Kharagpur, Kharagpur-721302. India. note that the experimental cell is also of general use in determining interfacial forces.

In particular, we are concerned with the experimental study of the generic constrained vapor bubble thermosyphon (CVBT) system presented in Figure 1 for a microgravity environment. For a completely wetting system, the liquid will coat all the walls of the chamber. For a finite contact angle system, some of the walls will have only a small amount of adsorbed vapor that changes the surface properties at the solid-vapor interface. Liquid will fill a portion of the corners in both cases. If temperature at End (2) is higher than End (1), because of an external heat source and sink, energy flows from End (2) to End (1) by conduction in the walls and by an evaporation, vapor flow, and condensation mechanism. The condensate flows from End (1) to End (2) because of the intermolecular force field that is a function of the film profile. A CVBT with a very small cross section has been called a micro heat pipe (Cotter, 1984; Peterson, 1992). The promise for high heat flux cooling has generated renewed interest in the application of micro heat pipes as efficient cooling devices. Potential applications are many, namely, cooling of electronic circuitry and thermal control of spacecraft. From a more fundamental point of view, the CVBT conveniently permits the study of interfacial transport concepts.

There is a "pressure jump" at the liquid-vapor interface, due to the anisotropic stress tensor near interfaces. For many 


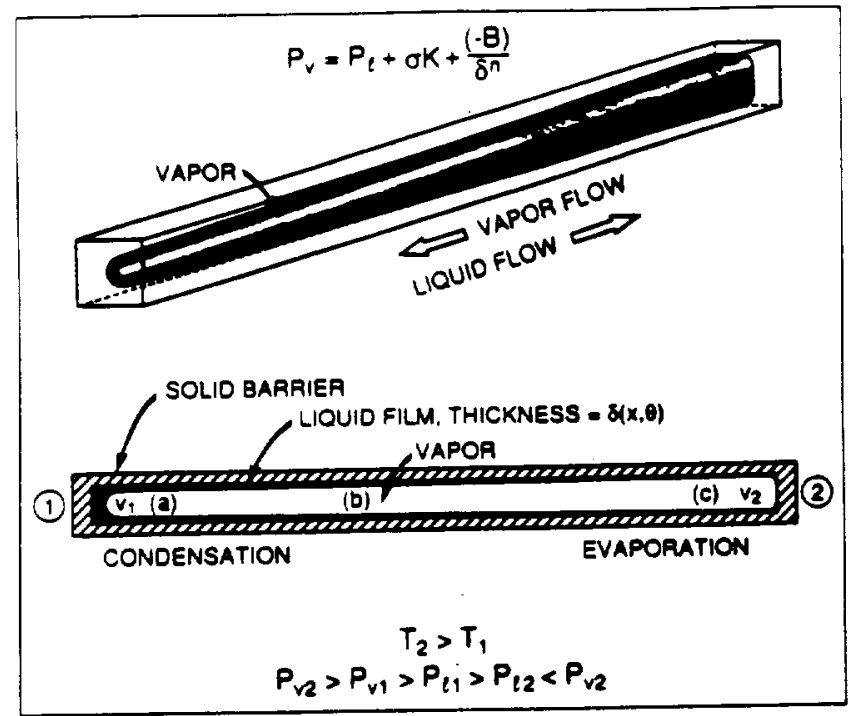

Figure 1. CVBT concept.

years, the classic Young-Laplace equation of capillarity has been successfully used to describe the pressure jump at a curved liquid-vapor interface. An example of its use to describe the fluid dynamics in a micro heat pipe is given in a publication by Wu and Peterson (1991). In this case, the pressure jump is a function of the liquid-vapor surface tension and the interfacial radius of curvature. However, near the liquid-solid interface, additional changes in the stress field within the liquid occur because of changes in the intermolecular force field due to solid molecules replacing liquid molecules. This leads to the augmented Young-Laplace model for the pressure jump at the liquid-vapor interface (Derjaguin and Kussakov, 1939; Potash and Wayner, 1972; Blake, 1975; Derjaguin and Churaev, 1976; Teletzke et al., 1976; Renk et al., 1978; Kamotani, 1978; Holm and Goplen, 1979; Moosman and Homsy, 1980; de Gennes, 1985; Truong and Wayner, 1987; DasGupta et al., 1993a). These long-range van der Waals forces have been found to be extremely important in that they lead to the concept of an extended evaporating meniscus (Potash and Wayner, 1972; Wayner et al., 1976). In a completely wetting evaporating system, a thin adsorbed film extends for a long distance beyond the classic equilibrium meniscus (such as Derjaguin et al., 1965; Wayner, 1991). The thin film controls the important processes of spreading and wetting. It forms a thin liquid bridge between the "classical menisci" formed in the corners of the chamber presented in Figure 1. Recently, Khrustalev and Faghri (1993) and Swanson and Peterson (1993) have used this type of model to analyze a micro heat pipe. Heat transfer from a stable evaporating thin film in the neighborhood of a contact line was analyzed by Brown et al. (1993). Swanson and Herdt (1992) have used the three-dimensional augmented Young-Laplace equation to develop a mathematical model describing the evaporating meniscus in a capillary tube. On the other hand. experimental results have been presented that question the validity of the augmented Young-Laplace equation: Using aqueous films, Lam and Schechter (1991) found deviations from the predicted profile obtained using a technique presented by Hirasaki (1990). Herein, we present the initial ex- perimental results of a study of the three-dimensional ex. tended meniscus in a contiguration of general importance to the microgravity environment: the constrained vapor bubble thermosyphon. Experimentally, we find that the augmented Young-Laplace equation is an excellent model for the force field at the solid-liquid-vapor interfaces for heptane and pentane menisci on quartz and tetradecane on SFL6.

The effects of both the liquid-vapor and liquid-solid interfaces on the effective pressure jump at the liquid-vapor interface of the extended two-dimensional meniscus have been modeled using the following augmented Young-Laplace equation:

$$
\begin{gathered}
P_{l}-P_{v}=\frac{\bar{B}}{\delta^{n}}-\sigma K \\
\frac{\bar{B}}{\delta^{n}}=\frac{B}{\delta^{4}}=-\Pi, \quad \delta \geq 40 \mathrm{~nm} \\
\frac{\vec{B}}{\delta^{n}}=\frac{\bar{A}}{\delta^{3}}=-\Pi \quad \delta \leq 20 \mathrm{~nm}
\end{gathered}
$$

where $P_{l}$ is the liquid pressure, $P_{u}$ is the vapor pressure, $\bar{A}$ is the Hamaker constant (negative for a completely wetting liquid), $B$ is the retarded dispersion constant for thicker films, $\delta(x)$ is the film thickness, $K$ is the curvature, and $\sigma$ is the surface tension. The first term on the righthand side of Eq. 1 is called the disjoining pressure, $-\Pi$, and it represents the change in the body force on the liquid due to the long-range van der Waals forces between the liquid and solid over a narrow range of thicknesses.

According to Eq. 1, the effective pressure in the liquid is reduced below that in the vapor by both capillarity and disjoining pressure in a completely wetting system. This leads to a reduction in the vapor pressure. However, the vapor pressure reduction can be offset by a temperature increase to obtain the vapor bubble thermosyphon presented in Figure 1. The chemical potential field is a function of the temperature and the effective liquid pressure that is a function of the shape of the liquid film. Therefore, the process is understood by measuring the temperature field and the liquid film shape.

\section{Exporiments}

A diagram of the cell and the experimental setup is shown in Figure 2. The readily available UV fused quartz cell (Type 607 Fluorimeter cell, NSG Precision Cells, Inc.) was essentially a small cuvette, selected to facilitate optical observation and measurement of the liquid meniscus. The cell was square ( $3 \mathrm{~mm} \times 3 \mathrm{~mm}$ inside) in cross section and about $40 \mathrm{~mm}$ long. The SFL6 glass cell was specially made from optically flat glass and is not readily available. The cell was cleaned after manufacturing and delivered in a closed container. The cells were claimed to be ready for spectroscopy. The cell was opened only inside a class -100 clean hood, rinsed repeatedly with pure ( $+99 \%$, as supplied by Aldrich Chemicals) and filtered test liquid before partially filling it. We examined the inside surface of the cell using high power microscopes and found them uniformly clean and sufficiently free of dust particles. As a result, we decided to use the quartz cell without any further wet cleaning. At the end of each experiment the 


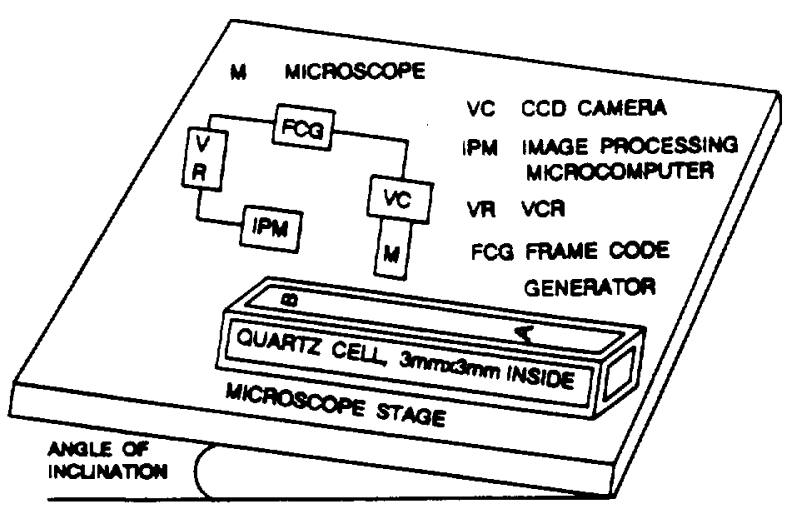

Flgure 2. Experimental setup.

The source of illumination was through the objective of the microscope.

cell was heated inside the clean hood at about $300^{\circ} \mathrm{C}$ for an hour and then rinsed with the new test liquid. We note that slight dehydration of the surface may take place during heating, but since we used only nonpolar liquids (alkanes), that would not affect adsorption on quartz (Gee et al., 1989). Using this procedure we were able to obtain a satisfactorily clean surface for experiments. The present study was conducted as a precursor to the nonisothermal CVBT experiments, mainly to develop the method for accurately measuring the liquid film thickness profile and to estimate the dispersion constant, in siru, at the start of the experiment. The isothermal results characterize the interfacial force field and the information is critical to the operation and the heat sink capability of the CVBT. The experimental cell was kept inclined to demonstrate the changes in shape of the meniscus as a result of externally imposed conditions (gravitational field, in this case). Examples of two cross sections of the cell that are a function of the system, location, gravitational field, volume of liquid, and cell size are presented in Figure 3.

The film thickness profile near the corners of the experimental cell was measured using image analyzing interferometry (IAI). A drawing of this section of the cell is presented in Figure 4. Interference phenomena were used to determine the profile of the capillary meniscus in the thickness range $\delta \geq 0.1 \mu \mathrm{m}$. A detailed description of the IAI techniques and hardware for film thickness profile measurement were presented in Sujanani (1990), Sujanani and Wayner (1991), and DasGupta et al. $(1991,1993 a, b)$ for a reflecting substrate. Blake (1975) used a transparent substrate in a related study.
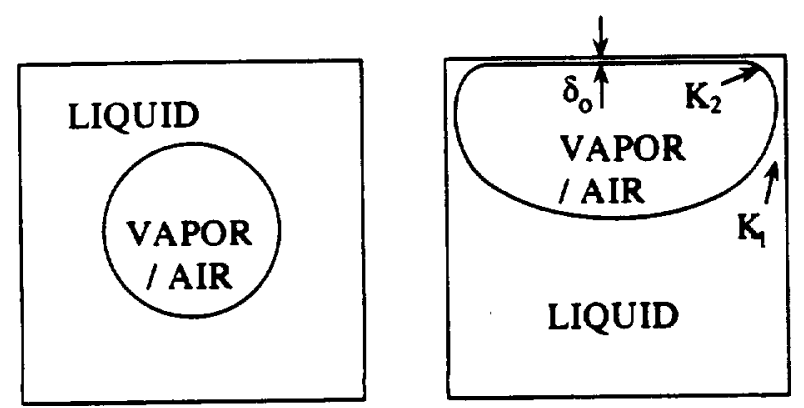

Figure 3. Example of the effect of gravity on the meniscus shape.

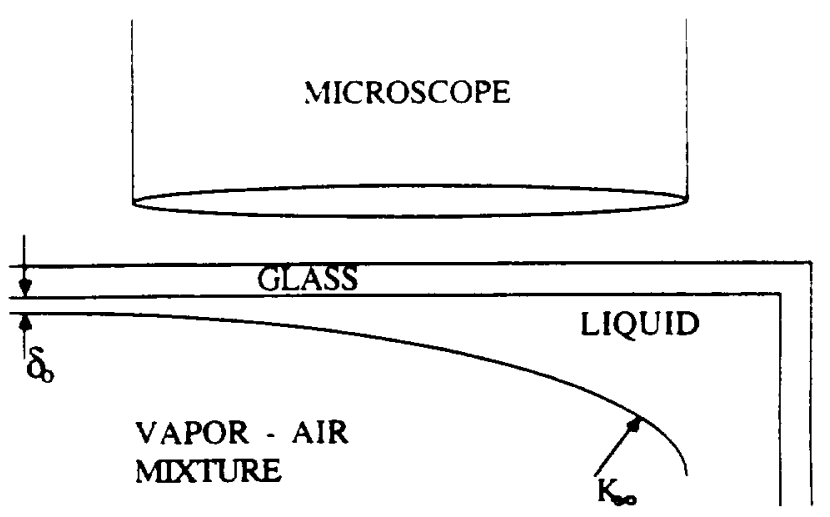

Figure 4. Location of microscope.

The image captured from the microscope through a CCD camera was digitized into 490 (vertical) $\times 512$ (horizontal) space pixels and assigned one of 256 possible gray values representing an intensity from 0 (black) to 255 (white). Thus each microscopic pixel acted as individual, simultaneous light sensors. For the present experimental setup, each pixel represented a diameter of $0.3 \mu \mathrm{m}$.

The cell was partiaily filled up with the liquid and placed on the microscope stage. The whole assembly was tilted with respect to the horizontal at two different angles in the two settings used in this study. The system was left to equilibrate with the surroundings for $3 \mathrm{~h}$ before taking any data. An optical interference pattern representing the thickness profile was readily observed (Figure 5). The pictures of the interference patterns formed at the corners of the cell at two specific points (A and B, as shown in Figure 2) for each angle setting were captured in the image processor, The exact locations of $A$ and $B$ varied with each system so that interference from small particles adhering to the surface, which caused nonparallel fringes in some regions, could be avoided. The vertical separation in the gravitational field between locations $A$ and B was $\Delta y$, which is given below. From each image a plot of the pixel gray value vs. distance was extracted. The gray value at each pixel was a measure of the reflectivity. As is evident from Figure 5 , the reflectivity underwent a cyclic change with increase in film thickness. The computer program scanned the peaks and valleys and filtered the noise from the real peaks/valleys. It then interpolated peak/valley envelopes and by analyzing the relative reflectivity of any pixel with respect to these (dark and light pixel envelopes) determined a film thickness at every pixel (DasGupta et al., 1991). The fact that the extended capillary meniscus merged smoothly to an adsorbed flat film was utilized to estimate the adsorbed film thickness from the gray value data and the peak/valley envelopes. The adsorbed film is obvious in the gray value plot since $G(x)<G_{\max }(x)$ in the region $x>50 \mu \mathrm{m}$. An estimation of the overall error associated with the measurements and data reduction procedures can be obtained by viewing the data in the comparison between theory and experiments presented below in Figure 11. We note that independent measurements of the curvature in the thick film region and the adsorbed film thickness in the flat region are needed.

Figures 6 and 7 are two examples of the measured film thickness profiles with heptane as the test liquid. Each picture was for a specific angle of inclination. In an isothermal 


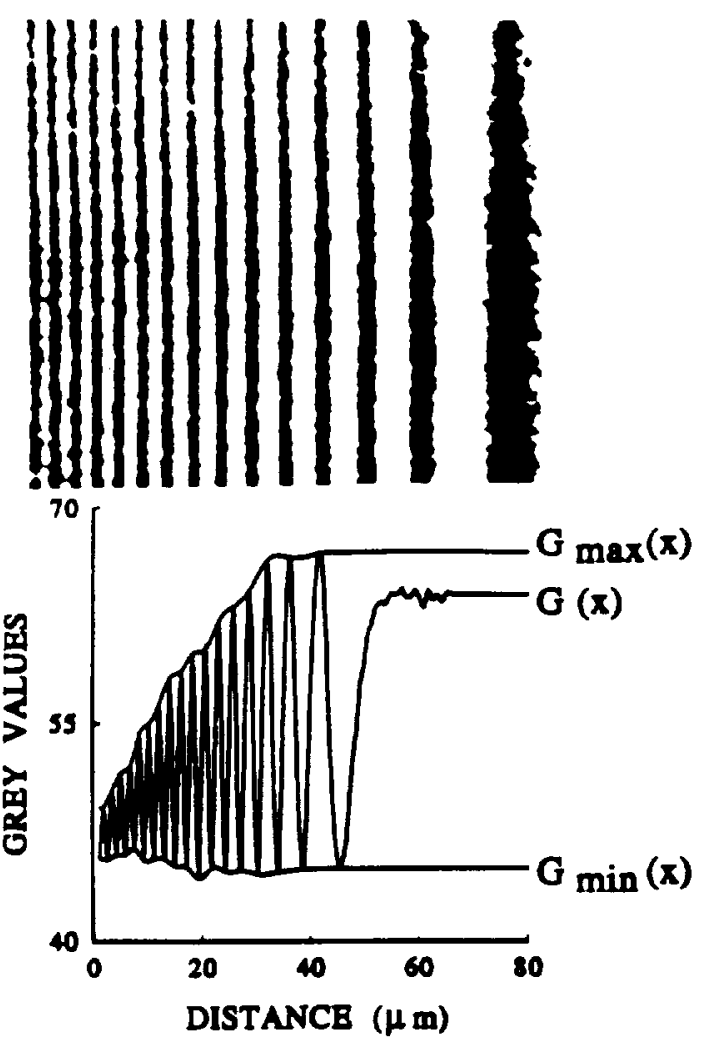

Figure 5. Interterence fringes and the gray value plot.

system of spreading liquid in an inclined cuvette, the curvature at a constant height in the gravitational field should remain constant in the region where dispersion forces can be neglected. So the film profile in this range approximated a parabola and a plot of $\delta^{1 / 2}$ vs. $x$ was a straight line. As can be seen from Figures 6 and $7, \delta^{1 / 2}$ vs. $x$ was nearly a straight line in all the cases, showing the proximity of the cases to isothermality. On the other hand, it is clear from these figures that the thickness profiles at point $B$ in each of these angle settings was steeper than at point $A$ as a result of higher hydrostatic pressures and subsequent higher curvatures. Again a comparison of Figures 6 and 7 demonstrates that, as expected, an increase in angle resulted in an increase in the film curvature. Plotting $\delta^{\sqrt{2}}$ vs. $x$, therefore, clearly illustrates the sensitivity of the film thickness shape to externally imposed conditions. This will not be obvious from a plot of $\delta$ vs. $x$. In addition, the adsorbed film thickness, $\delta_{0}$, is clearly seen in the region $x \geq 50 \mu \mathrm{m}$.

\section{Refractive index and contrast}

The contrast of the interference fringes of a liquid film formed on a solid substrate is a strong function of the refractive indices of the two media, more specifically, of the difference of the refractive indices of the solid and the liquid. Since we used optical techniques (microcomputer-enhanced video microscopy of the interference fringes) for the measurement of the film thickness profiles, the relative contrast of dark and bright fringes has a profound effect on the measurement capability and its accuracy. If the two refractive indices are

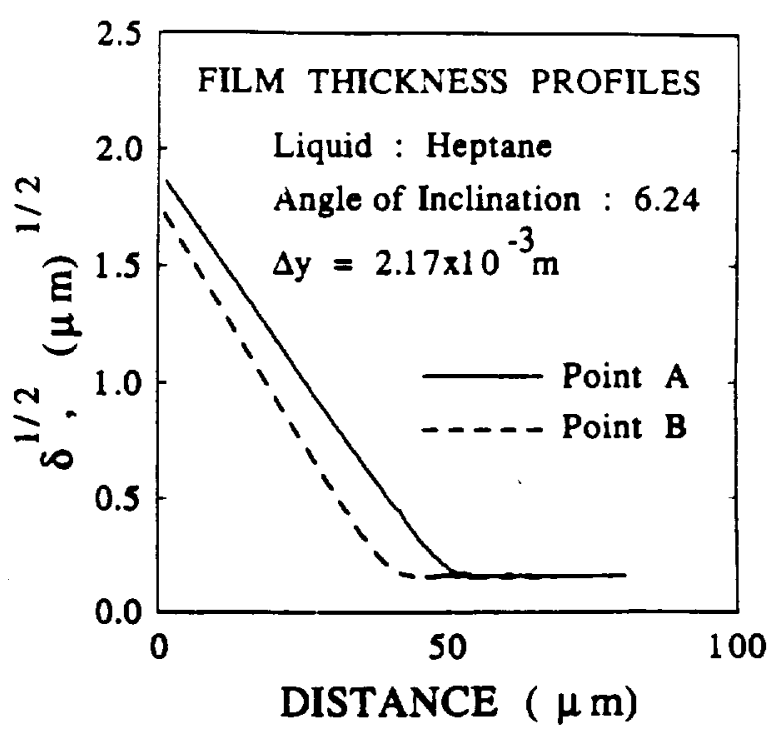

Figure 6. Film thickness profiles of Heptane at different positions (point $A$ and $B$ ) for an angle of inclination equal to $6.24^{\circ}$.

close to each other, it will be difficult to see the fringes and perform any image analysis due to the high noise in the captured picture. The following discussion gives a qualitative treatment of the problem.

The relative contrast, $C$, is defined in the following way,

$$
C=\left|\frac{R_{1}+R_{2}}{R_{1}-R_{2}}\right|^{2}
$$

Here $R_{1}$ and $R_{2}$ are the Fresnel coefficients that depend on the refractive indices of the solid, liquid and the vapor:

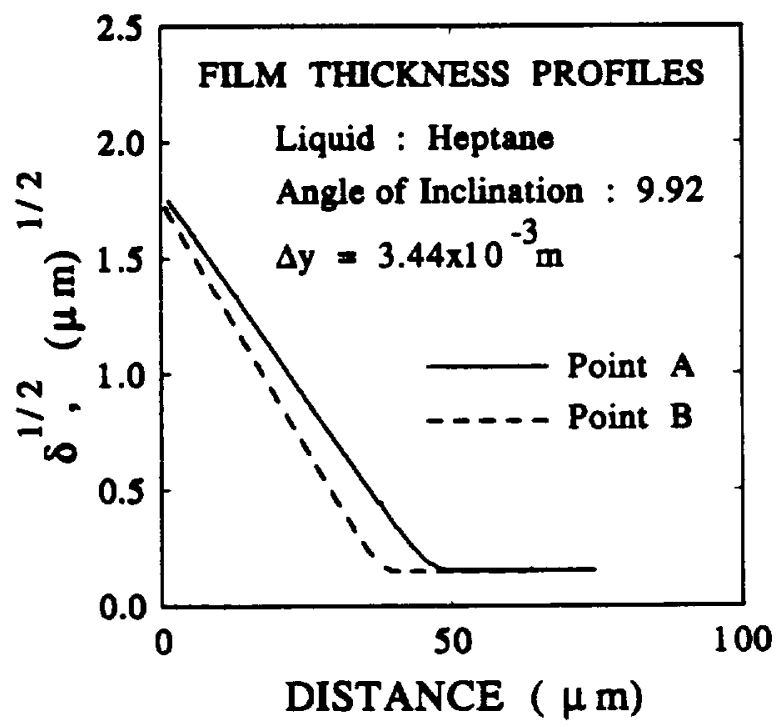

Figure 7. Film thickness profiles of Heptane at different positions (point $A$ and $B$ ) for an angle of inclination equal to $9.92^{\circ}$. 


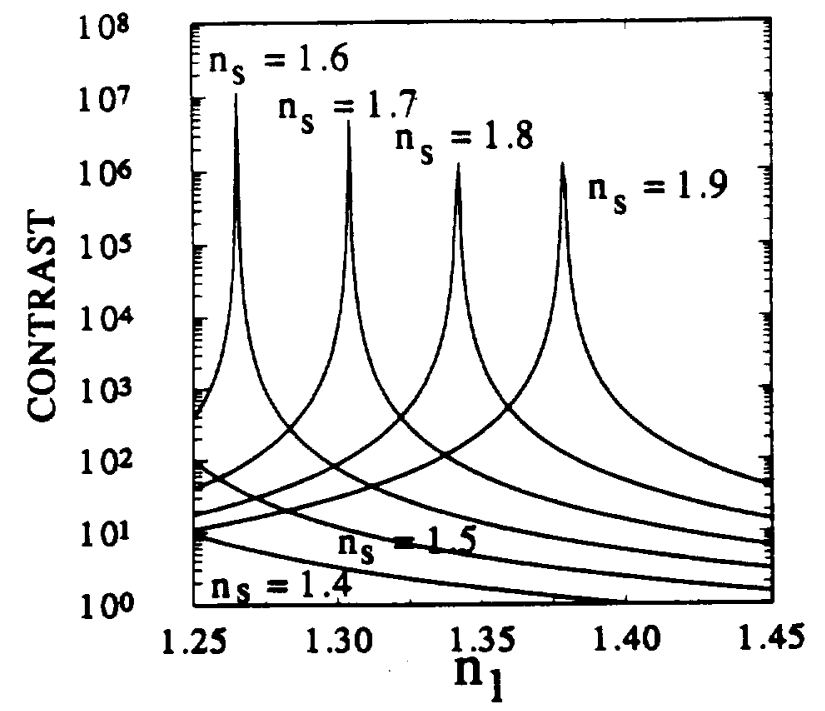

Figure 8. Relative contrast ve. liquid and solid refractive indices.

$$
R_{1}=\frac{n_{s}-n_{l}}{n_{s}+n_{l}}, \quad R_{2}=\frac{n_{l}-n_{v}}{n_{l}+n_{v}}
$$

Figure 8 is a plot of the relative contrast as a function of the solid and liquid refractive indices. As is evident from the figure, a drastic reduction in contrast takes place when the refractive index of a liquid being tested in the quartz cuvette is close to the refractive index of quartz $\left(n_{s}=1.5\right)$. We observed noticeable reduction in sharpness and contrast of the fringes between pentane $\left(n_{l}=1.358\right)$ and heptane $\left(n_{l}=1.387\right)$ menisci on quartz. But for tetradecane $\left(n_{l}=1.429\right)$ in our quartz CVBT, the fringes were blurred and the noise level was unacceptably high even after using image-enhancement techniques. Numerical values of $n, R$, and $C$ are given in Table 1 .

To solve this problem, we designed another CVBT with the same dimension but using a special high refractive index glass (SFL6, $n_{s}=1.8126$ at $548-\mathrm{nm}$ wavelength) to enhance the contrast and ensure the accuracy and ease of the optical measurements. We observed dramatic improvements in contrast (as can be expected from the results presented in Figure 8) and were able to conduct experiments with higher refractive index liquids (such as tetradecane).

\section{Theory}

The augmented Young-Laplace equation can be written for a point in the thicker portion of the meniscus, where the disjoining pressure effects are negligible and for another point

Table 1. Relative Contrast for Various Liquid-Solid Systems

\begin{tabular}{lccccccc}
\hline & & \multicolumn{2}{c}{$R_{1}$} & & \multicolumn{2}{c}{ Contrast $(C)$} \\
\cline { 3 - 6 } \cline { 5 - 7 } Liquid & $n_{1}$ & $n_{s}=1.5$ & $n_{s}=1.813$ & $R^{2}$ & $n_{s}=1.5$ & $n_{s}=1.813$ \\
\hline Pentane & 1.358 & 0.0497 & 0.1435 & 0.1518 & 3.895 & $1.255 E 03$ \\
Heptane & 1.387 & 0.0391 & 0.1331 & 0.1621 & 2.678 & $1.036 \mathrm{E} 02$ \\
Tetradecane & $\mathbf{1 . 4 2 9}$ & $\mathbf{0 . 0 2 4 2}$ & 0.1184 & 0.1766 & 1.737 & $2.573 \mathrm{E} 01$ \\
\hline
\end{tabular}

where both effects are present. For the isothermal nonevaporating (additional heat input, $Q=0$ ) cases considered here the liquid pressure will remain the same. irrespective of the position at a constant gravitational level. In our experiments, the following equation applies at a fixed hydrostatic head:

$$
\sigma K-\frac{B}{\delta^{4}}=\sigma K_{x}, \quad Q=0
$$

We note that the curvature at the thicker portion of the meniscus $\left(K_{x}\right)$ is nearly constant in the region studied (DasGupta et al., 1993b). In Eq. 4, the curvature $(K)$ can be expressed in the following form.

$$
K=\frac{\frac{d^{2} \delta}{d x^{2}}}{\left[1+\left(\frac{d \delta}{d x}\right)^{2}\right]^{3 / 2}}
$$

Using a simplified form of curvature. which is valid only if the square of the slope is small compared to one, Eq. 4 can be written in the following way:

$$
\sigma \frac{d^{2} \delta}{d x^{2}}-\frac{B}{\delta^{4}}=\sigma K_{x}
$$

For the isothermal experiments reported in this study the value of the maximum slope is 0.15 , and hence using the simplified form of curvature is justified. The following variables are introduced next to modify Eq. 6 to obtain Eq. 8:

$$
\begin{aligned}
& \eta=\frac{\delta}{\delta_{0}} \quad Z=x\left(\frac{K_{x}}{\delta_{0}}\right)^{1 / 2} \\
& \frac{d^{2} \eta}{d Z^{2}}+\left(\frac{-B}{\sigma K_{x} \delta_{0}^{4}}\right) \frac{1}{\eta^{4}}=1 .
\end{aligned}
$$

Equations 4-8 are valid for an equilibrium situation where no evaporation or condensation is taking place. A dimensionless variable, $a$, is defined next.

$$
\alpha^{4}=\frac{-B}{\sigma K_{x} \delta_{0}^{4}}
$$

Equation 8 can now be written as

$$
\frac{d^{2} \eta}{d Z^{2}}=1-\frac{\alpha^{4}}{\eta^{4}}
$$

For the equilibrium case, $Q=0$ and $\alpha=1$. Any values of $\alpha$ other than unity will signify deviation from the equilibrium situation that needs to be evaluated because of the sensitivity of the system to extremely small variations in the local temperature. The experimental section of the article and the subsequent discussion will present three experimental cases, which were very close to equilibrium. The results will demon- 
trate the utility of the model and the evaluation of the talues of $\alpha$

After multiplying both sides of Eq. 10 by $2 d \eta d Z$ and integrating $\left(C_{1}\right.$ is the constant of integration $)$.

$$
\left(\frac{d \eta}{d Z}\right)^{2}=2 \eta+\frac{2}{3} \frac{\alpha^{4}}{\eta^{3}}+C_{1}
$$

The boundary condition used for the completely wetting case is

$$
\text { For } \quad \eta=\alpha \quad \frac{d^{2} \eta}{d Z^{2}}=0=\frac{d \eta}{d Z} \text {. }
$$

Note that this can be viewed as both an artificial boundary condition for a nonequilibrium system for which $\alpha>1$ and the actual boundary condition for an equilibrium system for which $\alpha=1$.

Using the boundary condition, the slope of the meniscus can be expressed as

$$
\frac{d \delta}{d x}=-\left(K_{x} \delta_{0}\right)^{1 / 2} \sqrt{2 \eta+\frac{2}{3} \frac{\alpha^{4}}{\eta^{3}}-\frac{8}{3} \alpha} .
$$

Hence if the curvature at the thicker end of the meniscus, $K_{x}$, along with $B, \delta_{0}$, and $\sigma$ are known, the slope of the meniscus can be directly calculated as a function of the film thickness, using only the augmented Young-Laplace equation. The minus sign in Eq. 13 is indicative of the fact that for the reference frame selected, the meniscus slope should always be negative (film thickness decreases as distance increases). In a recent publication concerning a different experimental design, DasGupta et al. (1994) have demonstrated that the augmented Young-Laplace equation can be successfully used to evaluate the slope profiles for isothermal cases from known values of $K_{x}, B, \delta_{0}$, and $\sigma$.

For our present study we obtain the curvature in the thicker portion of the liquid meniscus in the following way. As can be seen from Figures 6 and 7, the isothermal film profile in the thicker portion of the meniscus approximated a parabola ( $\delta=a x^{2}$, with $a$ being a constant and the curvature equal to 2a). The values of $\delta^{1 / 2}$ and the corresponding $x$, for $\delta>0.5$ $\mu \mathrm{m}$. were fitted to a straight line using linear regression. The result was an excellent fit in each case ( $R^{2}$ values higher than $0.99)$ and from the regression routine the slope of the resulting straight line (equal to $a^{L / 2}$ ) and $K_{x}$ (equal to $2 a$ ) were readily obtained.

Herein we compare the slope of the meniscus obtained by numerical analysis of the experimental data for a system with unknown values of Hamaker cunstant (or dispersion constant, depending on the adsorbed film thickness) and the slope predicted by the augmented Young-Laplace equation (Eq. 13). The slope predicted by Eq. 13 is a function of $\alpha$ and the $\alpha$ corresponding to the closest match between these two slopes is selected to determine the value of the dispersion constant for the CVBT, in siru, for our isothermal experiments, as will be apparent in the next sections. Finally, we compare the experimentally obtained values of the dispersion constants and the disjoining pressures with the prediction from the DLP theory (Dzyaloshinskii et al. IGhll for wur oxperimental system.

\section{Results and Discussion}

The objective of this work is to characterize the interfacial force field by evaluating the dispersion constant. in situ, tor the solid-liquid-vapor system. Once the film thickness profile. including the adsorbed film thickness. $\delta_{11}$, were obtained. the curvature at the thicker end of the meniscus. $K_{\leftarrow}$. was determined. Equation 13 was then used to calculate the slope of the thickness profiles as a function of $\alpha$ and the $\alpha$ corresponding to the closest match between the experimental slope, obtained by numerical differentiation of a cubic spline fitted to the data, and Eq. 13 was used to determine the dispersion constant for the specific liquid-solid system.

Figures 9 and 10 show two pictures of the close match between the experimental slope and the slope obtained by the solution of the augmented Young-Laplace equation. The solid line represents the slope obtained by numerical differentiation of the data, whereas the triangles represent the solution of the augmented Young-Laplace equation for the specific value of $\alpha$ at every tenth data point. In Table 2 the results for pentane, which are a strong function of the value of $\alpha$, are summarized. Similar results were also obtained for heptane.

The experimental values of the dispersion constants for the systems are presented in Table 3 . The dispersion constants are calculated from the known values of $\alpha$ corresponding to the minimum error. The values of $B$ for each liquid are reasonably close to each other. We also calculated the dispersion force and the theoretical values of $B$ from the DLP theory (Lifshitz, 1955; Dzyaloshinskii et al., 1961) for quartzpentane-vapor and quartz-heptane-vapor systems. To calculate the dispersion force from the DLP theory, the same methodology presented by Truong and Wayner (1987) and Gee et al. (1989) is used. The dielectric functions of the liq-

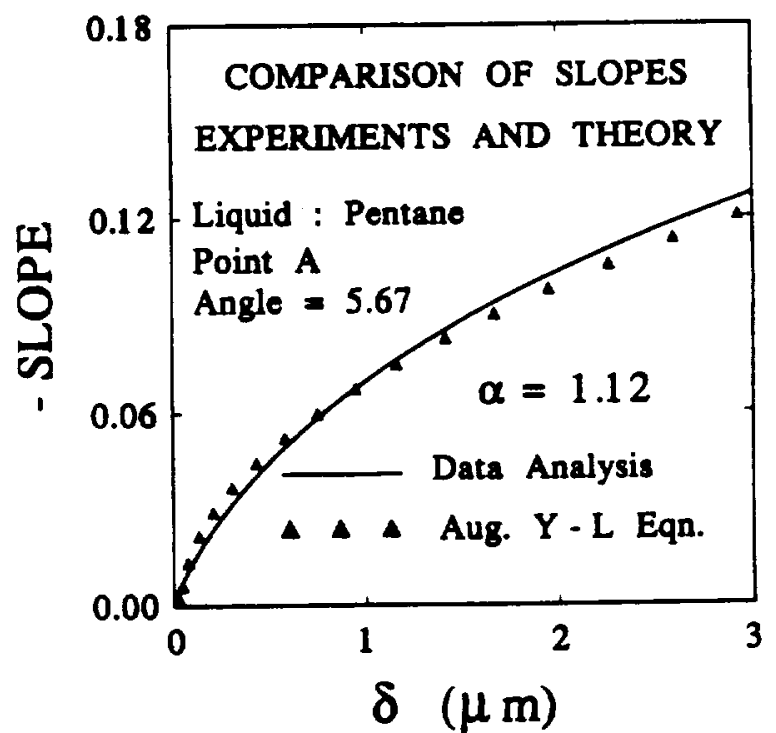

Figure 9. Experimental slope vs. that obtained from the augmented Young-Laplace equation (Eq. 13). 


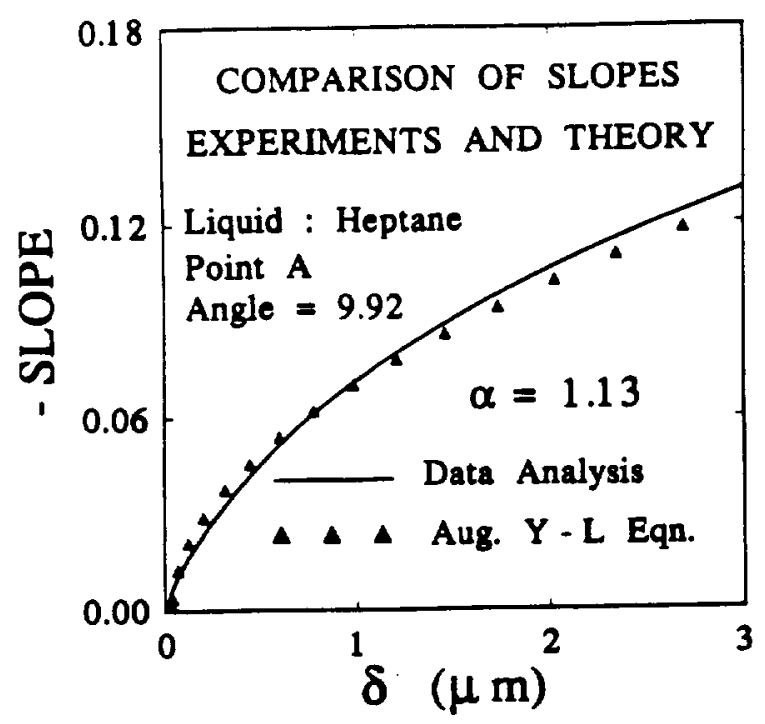

Flgure 10. Experimental slope ve. that obtained from the augmented Young-Laplace equation (Eq. 13).

uids and substrates are obtained from spectroscopic optical data given in Table 4 as described by Hough and White (1980). The results are presented in Table 3 and in Figure 11. Figure 11 is a plot of the disjoining pressure as a function of the film thickness for pentane and heptane wetting on quartz, and for tetradecane on SFL6. The solid line is from DLP theory, whereas the symbols are from the experiments. Since the values of the dispersion forces for the two alkanes on quartz, as predicted by the DLP theory, are about the same, only the theoretical values for pentane are presented. Both Table 3 and Figure 11 clearly demonstrate that our experimentally obtained values of $B$ and the disjoining pressures for the two liquids in the quartz cell are close to those predicted from the exact DLP theory. Comparable results for tetradecane on SFL6 are presented in Figure 11 and Table 5. However, the DLP calculations for SFL6 are based on incomplete optical

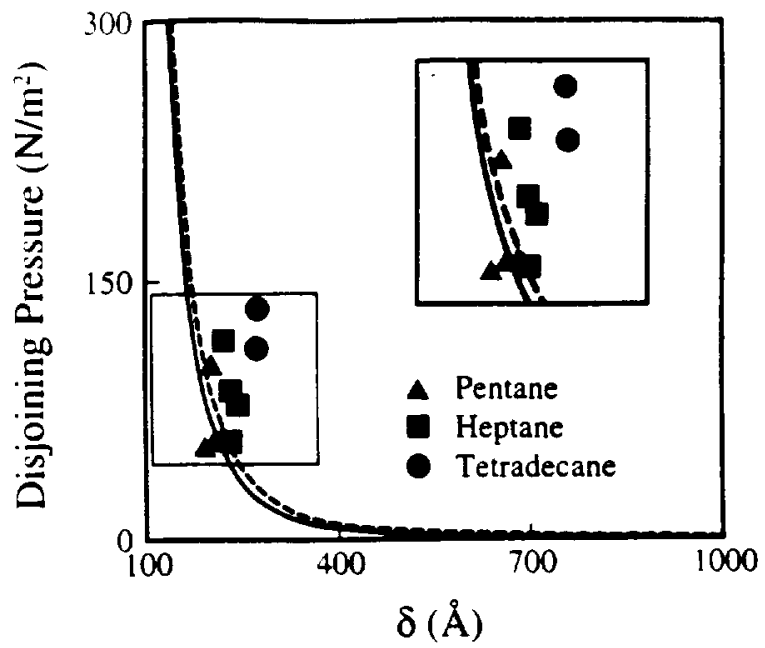

Flgure 11. DLP theory for an Ideal surface va. experiments for the adsorption of pentane and heptane on quartz (-) and tetradecane on SFL6 based on UV data only (-- - ).

data. We note that the DLP theory predicts the dispersion forces for an ideal system. whereas the surface energies of real surfaces can be quite different because of the presence of impurities or adsorbed water layers. The importance of an in situ force field characterization technique cannot therefore be overemphasized.

Similar results were obtained by Gee et al. (1989) from adsorption studies of $\boldsymbol{n}$-alkanes on quartz for high disjoining pressure regimes ( $\delta \leq 40 \AA$ ) and by Blake (1975), who measured the disjoining pressure as a function of film thickness for $n$-octane and $n$-decane on $\alpha$-alumina. Blake's techniques demonstrated that Lifshitz theory correctly predicts the results in the low disjoining pressure regimes ( $\delta$ was between $250 \AA$ and $800 \AA$ ), whereas our present study is concerned with adsorbed film thicknesses of about $200 \AA$. For completeness, we also note that recent studies by Beaglehole et al.

Table 2. Characteristics of Pentane Meniscus

\begin{tabular}{|c|c|c|c|c|c|c|c|c|}
\hline & \multicolumn{4}{|c|}{ Angle of Inclination $=5.67$} & \multicolumn{4}{|c|}{ Angle of Inclination $=9.92^{\circ}$} \\
\hline & $\begin{array}{c}\delta_{0} \\
(\mathrm{~nm})\end{array}$ & $\begin{array}{c}K_{x} \times 10^{-3} \\
\left(\mathrm{~m}^{-1}\right)\end{array}$ & $\alpha$ & $\begin{array}{c}B \\
(J \cdot m)\end{array}$ & $\begin{array}{c}\delta_{0} \\
(\mathrm{~nm})\end{array}$ & $\begin{array}{c}K_{x} \times 10^{-3} \\
\left(\mathrm{~m}^{-1}\right)\end{array}$ & $\alpha$ & $\begin{array}{c}B \\
(J \cdot m)\end{array}$ \\
\hline $\begin{array}{l}\text { Point A } \\
\text { Point B }\end{array}$ & $\begin{array}{l}22.0 \\
20.0\end{array}$ & $\begin{array}{l}2.512 \\
3.147\end{array}$ & $\begin{array}{l}1.12 \\
1.04\end{array}$ & $\begin{array}{l}1.43 \times 10^{-29} \\
0.91 \times 10^{-29}\end{array}$ & $\begin{array}{l}19.0 \\
20.0\end{array}$ & $\begin{array}{l}3.044 \\
4.186\end{array}$ & $\begin{array}{l}1.04 \\
1.12\end{array}$ & $\begin{array}{l}0.72 \times 10^{-29} \\
1.63 \times 10^{-29}\end{array}$ \\
\hline
\end{tabular}

Table 3. Experimental and Theoretically Calculated Values of $B(\delta)$ for Pentane and Heptane on Quarts

\begin{tabular}{|c|c|c|c|c|c|c|c|}
\hline \multicolumn{4}{|c|}{$n$-pentane } & \multicolumn{4}{|c|}{$n$-heptane } \\
\hline $\begin{array}{c}\text { Angle } \\
\left({ }^{\circ}\right)\end{array}$ & $\begin{array}{c}\delta_{0} \\
(\mathrm{~nm})\end{array}$ & $\begin{array}{l}B_{\text {expl }} \\
(\mathrm{J} \cdot \mathrm{m})\end{array}$ & $\begin{array}{l}B_{D L P} \\
(\mathrm{~J} \cdot \mathrm{m})\end{array}$ & $\begin{array}{c}\text { Angle } \\
\left({ }^{\circ}\right)\end{array}$ & $\begin{array}{c}\delta_{0} \\
(\mathrm{~nm})\end{array}$ & $\begin{array}{c}B_{\text {expt }} \\
(\mathrm{J} \cdot \mathrm{m})\end{array}$ & $\begin{array}{l}B_{O L P} \\
(\mathrm{~J} \cdot \mathrm{m})\end{array}$ \\
\hline \multirow{2}{*}{5.67} & 22.0 & $1.43 \times 10^{-29}$ & $1.14 \times 10^{-29}$ & \multirow{2}{*}{6.24} & 24.0 & $2.60 \times 10^{-29}$ & $1.07 \times 10^{-29}$ \\
\hline & 20.0 & $0.91 \times 10^{-29}$ & $1.11 \times 10^{-29}$ & & 23.0 & $1.57 \times 10^{-29}$ & $1.05 \times 10^{-29}$ \\
\hline \multirow{2}{*}{9.92} & 19.0 & $0.72 \times 10^{-29}$ & $1.09 \times 10^{-29}$ & \multirow{2}{*}{9.92} & 23.0 & $2.41 \times 10^{-29}$ & $1.05 \times 10^{-29}$ \\
\hline & 20.0 & $1.63 \times 10^{-29}$ & $1.11 \times 10^{-29}$ & & 22.0 & $2.71 \times 10^{-29}$ & $1.04 \times 10^{-29}$ \\
\hline
\end{tabular}


Table 4. Optical Constants L'sed the Calculation of Dispersion Force from DLP Theory

\begin{tabular}{lcccc}
\hline & $C_{u i}$ & $\begin{array}{c}\omega_{i} \\
(\mathrm{rad} / \mathrm{s})\end{array}$ & $C_{i R}$ & $\begin{array}{c}\omega_{/ R} \\
(\mathrm{rad} / \mathrm{s})\end{array}$ \\
\hline Pentane & 0.819 & $1.877 \times 10^{16}$ & 0.025 & $5.54 \times 10^{14}$ \\
Heptane & 0.898 & $1.870 \times 10^{15}$ & 0.025 & $5.54 \times 10^{14}$ \\
Tetradecane & 1.011 & $1.846 \times 10^{16}$ & 0.025 & $5.54 \times 10^{14}$ \\
Quartz & 1.359 & $2.032 \times 10^{16}$ & 1.93 & $2.093 \times 10^{14}$ \\
SFL6 & 2.0953 & $1.191 \times 10^{16}$ & & \\
\hline
\end{tabular}

(From Hough and White. 1980).

(1991) and Beaglehole and Christenson (1992) demonstrate the limitations of the DLP theory in predicting the interactions in very thin liquid films (below $20 \AA$ ), and their results suggest that structural effects are present in some adsorbed films at room temperature, provided the substrates are smooth and homogeneous.

\section{Effects of evaporation on $\alpha$}

The augmented Young-Laplace equation can be written in the following way

$$
\phi=\frac{P_{1}-P_{v}}{\bar{A} / \delta_{0}^{3}}=\frac{1}{\eta^{3}}+\frac{1}{\alpha^{3} K_{x}} \frac{d^{2} \delta}{d x^{2}}
$$

where $\phi$ is the dimensionless pressure. Note that we are using a different expression for the disjoining pressure (equal to $\left.\bar{A} / \delta_{0}^{3}\right)$, and hence the nondimensional augmented Young-Laplace equation is expressed in a slightly different way with $B / \delta_{0}$ substituted by $\bar{A}$ in the expression for $\alpha$. Nevertheless, all the physical significances of $\alpha$ remain unchanged. This was done because we will be using some experimental data from another study and the magnitude of the adsorbed film thickness in that study warranted the use of the modified Hamaker constant instead of the dispersion constant.

In the thicker portion of the meniscus (large $\eta$ ), the previous equation can be simplified to obtain

$$
\alpha^{3}=\frac{1}{\phi}, \quad \eta \rightarrow \infty
$$

For an ideal equilibrium case, $\phi=1$ and there is no evaporation, condensation and flow in the meniscus. Whereas, for a nonequilibrium case, $\phi$ deviates from 1 and a larger than unity value of $\alpha$ is obtained when evaporation occurs. Since $\phi$ in Eq. 15 is equal to the ratio of the pressure jump across the liquid-vapor interface at large $\eta$ to the pressure jump at

Table 5. Experimental and Theoretically Calculated Values of $B(\delta)$ for Tetradecane on SFL6

\begin{tabular}{ccccc}
\hline \multicolumn{5}{c}{ Tetradecane } \\
\hline $\begin{array}{c}\text { Angle } \\
\left({ }^{\circ}\right)\end{array}$ & $\begin{array}{c}\delta_{0} \\
(\mathrm{~nm})\end{array}$ & $\alpha$ & $\begin{array}{c}B_{\text {expt }} \\
(\mathrm{J} \cdot \mathrm{m})\end{array}$ & $\begin{array}{c}B_{\text {DLP }} \\
(\mathrm{J} \cdot \mathrm{m})\end{array}$ \\
\hline \multirow{2}{*}{6.88} & 27.0 & 1.14 & $5.87 \times 10^{-29}$ & $1.60 \times 10^{-29}$ \\
& 27.0 & 1.15 & $7.12 \times 10^{-29}$ & $1.60 \times 10^{-29}$ \\
\hline
\end{tabular}

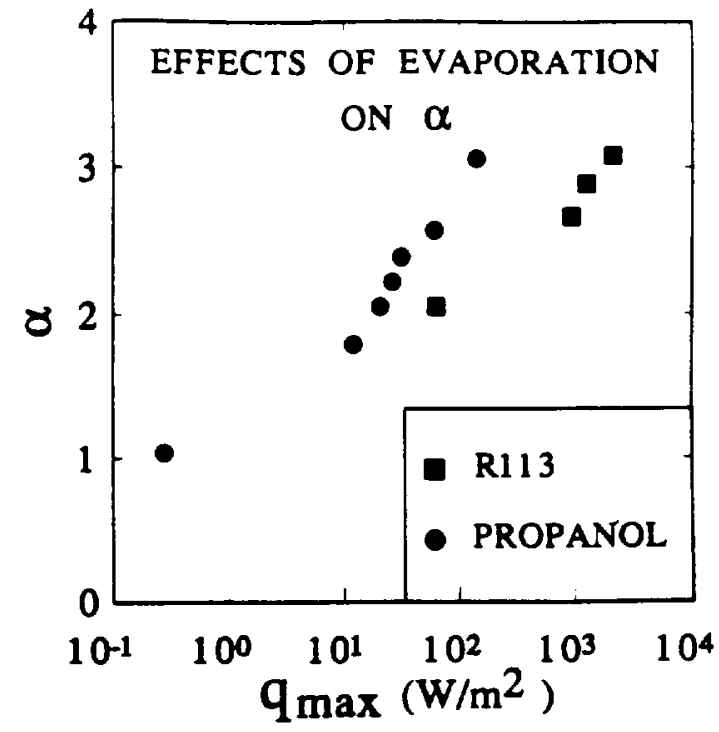

Figure 12. Effects of evaporation on the values of $\alpha$ for propanol and R113 menisci.

$\eta=1$, we find that $(1-\phi \eta)$ is a measure of the average dimensionless shear stress at the liquid-solid interface due to liquid flow. The theoretical value of $\phi$ for a given evaporative heat flux can be obtained using the equations presented by DasGupta et al. (1993b). Therefore, we can connect the very small value of $\alpha$ found herein with an extremely small amount of phase change.

We reanalyzed the data for some of the nonisothermal (and a few isothermal) cases observed in a different experimental setup designed to study evaporation. In those experiments a capillary feeder was used to study and model evaporating menisci of various liquids on a silicon substrate (DasGupta et al., 1993b). The values of $\delta_{0}$ were accurately measured using ellipsometry. A theoretical model based on the augmented Young-Laplace equation and modified Kelvin-Clapeyron equation was used. The numerical solution of the proposed model and comparison of the results with experimental data were used to characterize the system and to obtain the dimensionless pressure difference $(\phi)$ and maximum evaporative heat flux. The maximum heat flux data were presented in a related publication as a function of the interfacial temperature difference (DasGupta et al., 1994). Herein we utilize these results to calculate the values of $\alpha$ to demonstrate the sensitivity of $\alpha$ to evaporation.

Figure 12 is a plot of $\alpha$ as a function of maximum evaporative heat flux for nonequilibrium propanol and R113 menisci. The figure clearly demonstrates that $\alpha$ increases with an increase in evaporation (departure from equilibrium). The first points for both propanol and R113 data are interesting to note. For both of these cases the heater was off, but as the values of $\alpha$ show, the case for propanol was very close to isothermality ( $\alpha=1.037$ with a very small value for the heat flux), whereas the case for R113 is farther from equilibrium $(\alpha=2.05)$ due to uncontrolled environmental conditions. The respective temperature differences between the solid and vapor were found to be approximately $10^{-5}$ and $10^{-4} \mathrm{~K}$. This is an example of the sensitivity of the whole process. We also note that a small change in the value of $\alpha$ can signify a large 
change in the evaporative heat flux. A value of $\alpha$ greater than one denotes the presence of a curvature gradient in the flow field and shear stress due to the resulting flow.

The most important point of this study is the demonstration that the dispersion constants for a constrained vapor bubble thermosyphon can be evaluated in situ at the start of the experiments. This is a definite improvement over previous studies of micro heat pipes where little attention was given to the values of dispersion constants that are a strong function of the previous history of the substrate used in the experiments. These constants characterize the interfacial force field and are profoundly important in the basic understanding of the operation and performance of a micro heat pipe and other small thermosyphons (which includes the microlayer in boiling).

\section{Conclusions}

- The use of IAI in conjunction with a CVBT in the earth's gravitational field under isothermal conditions was demonstrated.

- The accuracy of the results are a function of the difference between the refractive indices of the solid and the liquid.

- Procedures to measure the dispersion constant in situ for the vapor-liquid-solid system were developed and good agreement between theoretical and experimental values of the dispersion constant was obtained.

- The augmented Young-Laplace equation accurately models the change in the effective pressure at the liquid-vapor interface.

- The sensitivity of the process and the physical significance of the parameter $\alpha$ in relation to the closeness to equilibrium were demonstrated.

\section{Acknowledgments}

This material is based on work supported by the National Aeronautics and Space Administration under grant NAG3-1399. Any opinions, findings, and conclusions or recommendations expressed in this publication are those of the authors and do not necessarily reflect the view of NASA.

\section{Notation}

$$
\begin{aligned}
x & =\text { distance, } m \\
Z & =\text { dimensionless distance, Eq. } 7 \\
\eta & =\text { dimensionless film thickness, } \delta / \delta_{0} \\
s & =\text { solid } \\
Y-L & =\text { Young-Laplace equation }
\end{aligned}
$$

\section{Literature Cited}

Beaglehole, D., E. Z. Radlinska, B. W. Ninham, and H. K. Christenson, "Inadequacy of Lifshitz Theory for Thin Liquid Films," Phys. Rev. Lett., God 16), 2084 (1991).

Beaglehole, D., and H. K Christenson, "Vapor Adsorption on Mica and Silicon: Entropy Effects, Layering and Surface Forces,"J. Phys. Chem., 96, 3395 (1992).

Blake, T. D., "Investigation of Equilibrium Wetting Films of n-alkanes on a-alumina." J. Chem. Soc., Farad Trans. 1, 71, 192 (1975).

Brown, J. R., W. S. Chang, K. P. Halliman, and H. C. Chebaro. "Heat Transfer from Stable Evaporating Thin Films in the Neighborhood of a Contact Line," Proc. Heat Transfer Conf., 93-HT-4, Atlanta, GA (1993).

Cotter, T. R., "Principles and Properties of Micro Heat Pipes," Phoc. Int. Heat Pipe Conf., Tsukuba, Japan, p. 328 (1984).
DasGupta. S., J. A. Schonberg, 1. Y. Kim. and P. C. Wayner. Jr Lse of the Augmented Young-Laplace Equation to Model Equilibrium and Evaporating Extended Menisci," J. Colloud Intert. Sct. 157. 332 (1993a).

DasGupta. S.. J. A. Schonberg, and P. C. Wayner. Jr., " Investigation of an Evaporating Ertended Meniscus Based on the Augmented Young-Laplace Equation," J. Heat Transfer, 115. 201 (1993b).

DasGupta, S., M. Sujanani, and P. C. Wayner, Jr., "Microcomputer Enhanced Optical Investigation of an Evaporating Liquid Film Controlled by a Capillary Feeder." Proc. World Conf. Expenmental Heat Transfer, Fluid Mechanics \& Thermodynamics. J. F. Keffer, et al., ed., Elsevier. New York. p. 361 (1991).

DasGupta, S., I. Y. Kim, and P. C. Wayner. Jr.. "Use of the KelvinClaypeyron Equation to Model an Evaporating Microfilm,"J. Heat Transf., 116, 1007 (1994).

deGennes, P. G.. "Wetting: Statics and Dynamics," Rev. Mod. Phys., 57, 289 (1985).

Derjaguin, B. V., and M. M. Kussakov, Acia Phys. LRSS, 10, 25 (1939).

Derjaguin, B. V., S. V. Nerpin, and N. V. Churaev. "Effect of Film Transfer upon Evaporation of Liquids from Capillaries." Bull. Rilem, No. 29, 93 (1965).

Derjaguin, B. V., and N. V. Churaev, "The Definition of Disjoining Pressure and its Importance in the Equilibrium and Flow of Thin Films," Colloid J. USSR, 38, 438 (1976).

Dzyaloshinskii, I. E., E. M. Lifshitz, and L. P. Pitaevskii, "The General Theory of van der Waals Forces," Ad. Phys., 10. 165 (1961).

Gee, M. L., T. W. Healy, and L. R. White, "Ellipsometric Studies of Alkane Adsorption on Quartz," J. Colloid Interface Sci., 131(1), 18 (1989).

Hirasaki, G. J., in Interfacial Phenomena in Petroleum Recovery, N. R. Morrow, ed., Marcel Dekker, New York (1990).

Holm, F. W., and S. P. Goplen, "Heat Transfer in the Meniscus Thin-Film Transition Region," J. Heat Transf., 101, 543 (1979).

Hough, D. B., and L. R. White, "The Calculation of Hamaker Constants from Lifshitz Theory with Applications to Wetting Phenomena," Adv. Coll. Interf. Sci. 14, 3 (1980).

Huh, C., and L. E. Scriven, "Hydrodynamic Model of Steady Movement of a Solid/Liquid/Fluid Contact Line," J. Colloids Interf. Sci. 35, 85 (1971).

Kamotani, Y., "Evaporator Film Coefficients of Grooved Heat Pipes," Proc. Int. Heat Pipe Conf., Palo Alto, CA (1978).

Khrustalev, D., and A. Faghri, "Thermal Analysis of a Micro Heat Pipe," Proc. Nat. Heat Transfer Conf., Atlanta, GA (1993).

Lam, A. C., and R. S. Schechter, "A Note on the Transition Region Connecting Thin to Thick Films," J. Colbids Interf. Sci. 146, 206 (1991).

Lifshitz, E. M., "Theory of Molecular Attractions Between Solid Bodies," J. Exp. Theor. Phys. USSR, 29, 94 (1955).

Miller, C. A., and E. Ruckenstein, "The Origin of Flow during Wetting of Solids," J. Colloids Interf. Sci. 48, 368 (1974).

Moosman, S., and S. M. Homsy, "Evaporating Menisci of Wetting Fluids," J. Colloids Interf. Sci. 73, 212 (1980).

Peterson, G. P., "Overview of Micro Heat Pipe Research and Developments," Appl. Mech. Rev., ASME, 45(5), 175 (1992).

Potash, M., Jr., and P. C. Wayner, Jr., "Evaporation from a Two-Dimensional Extended Meniscus," Int. J. Heat Mass Transf., 15, 1851 (1972).

Renk, F., P. C. Wayner, Jr., and G. M. Homsy, "On the Transition between a Wetting Film and a Capillary Meniscus," $J$. Colloid Interf. Sci., 67, 408 (1978).

Sujanani. M., "Interfacial Phenomena in an Evaporating Thin Film," PhD thesis, Rensselaer Polytechnic Institute, Troy, NY (1990).

Sujanani, M., and P. C. Wayner, Jr., "Microcomputer-Enhanced Optical Investigation of Transport Processes with Phase Change in Near Equilibrium Thin Liquid Films," J. Colloid Interface Sci, 143, 472 (1991).

Swanson, L. W., and G. C. Herdt, "Model of the Evaporating Meniscus in a Capillary Tube," J. Heat Transfer, 114, 434 (1992).

Swanson, L. W., and G. P. Peterson, "The Interfacial Thermodynamics of the Capillary Structures in Micro Heat Pipes," Proc. Nat. Heat Transfer Conf., Aug. 8-11, Atlanta, GA (1993).

Teletzke, G. F., L. E. Scriven, and H. T. Davis, "How Liquids Spread on Solids," Chem. Eng. Comm., 55, 41 (1987).

Truong, J. G., and P. C. Wayner, Jr., "Effect of Capillary and .Van 